\title{
IDENTIFICANDO BARREIRAS E CUMPRIMENTO DE METAS PARA PRÁTICA DE AUTOCUIDADO EM PESSOA COM DIABETES TIPO 2
}

\author{
Elisângela da Cruz Vargas¹, Sumaya Giarola Cecilio², Célia Luciana Guedes Barbosa Brasil'1, Heloisa de Carvalho Torres ${ }^{3}$
}

${ }^{1}$ Discente de Enfermagem. Universidade Federal de Minas Gerais. Belo Horizonte, MG, Brasil.

${ }^{2}$ Enfermeira. Especialista em Auditoria em Saúde. Mestranda em Enfermagem. Universidade Federal de Minas Gerais. Belo Horizonte, MG, Brasil.

${ }^{3}$ Enfermeira. Pós Doutora em Enfermagem. Docente do Departamento de Enfermagem da Universidade Federal de Minas Gerais. Belo Horizonte, MG, Brasil.

RESUMO: Objetivou-se identificar barreiras e o cumprimento de metas para o desenvolvimento das práticas de autocuidado em usuários com a condição crônica do diabetes Mellitus tipo 2. Estudo descritivo exploratório, realizado com 30 usuários, mediante visita domiciliar, em três unidades básicas de saúde de Belo Horizonte, Minas Gerais durante o ano de 2014. As barreiras e metas citadas foram analisadas mediante frequência relativa e agrupadas em categorias. $63,1 \%$ dos usuários apresentaram dificuldades relacionadas a comportamentos psicossociais e culturais, $16,5 \%$ a ambientais e estruturais, 3,3\% econômicos, $6,6 \%$ relacionaram a outros fatores e ainda $10 \%$ não consideraram ter dificuldades relevantes para cuidar da sua saúde. $60 \%$ dos usuários demonstraram disposição para planejamento e cumprimento de metas. Conclui-se que o usuário é capaz de buscar soluções para as barreiras e tomar decisões informadas, adequadas à sua saúde e contexto de vida, buscando soluções para o enfrentamento de barreiras nas práticas de autocuidado em diabetes.

DESCRITORES: Educação em saúde; Diabetes Mellitus; Autocuidado; Enfermagem.

\section{IDENTIFYING BARRIERS AND TARGET COMPLIANCE FOR SELF-CARE IN TYPE 2 DIABETES PATIENTS}

ABSTRACT: The objective was to identify barriers and compliance with targets for the development of self-care practices in chronic patients suffering from type 2 Diabetes Mellitus. Descriptive and exploratory study involving 30 users, undertaken through home visits at three primary health care services in Belo Horizonte, Minas Gerais in 2014 The barriers and targets cited were analyzed using relative frequencies and grouped into categories. $63.1 \%$ of the users presented difficulties related to psychosocial and cultural behaviors, $16.5 \%$ to environmental and structural behaviors, $3.3 \%$ economic behaviors, $6.6 \%$ related to other factors and $10 \%$ did not consider they had any relevant difficulties to take care of their health. Sixty percent of the users are capable of seeking solutions for the barriers and of making informed decisions that are appropriate to their health and life context, trying to find solutions to cope with barriers in diabetes self-care practices.

DESCRIPTORS: Health education; Diabetes Mellitus; Selfcare; Nursing.

\section{IDENTIFICANDO OBSTÁCULOS Y CUMPLIMIENTO DE METAS PARA PRÁCTICA DE AUTOCUIDADO EN PERSONA CON DIABETES TIPO 2}

RESUMEN: Fue objetivo del estudio identificar obstáculos y el cumplimiento de metas para el desarrollo de las prácticas de autocuidado en usuarios con la condición crónica del diabetes Mellitus tipo 2. Estudio descriptivo exploratorio, realizado con 30 usuarios, por visita domiciliar, en tres unidades básicas de salud de Belo Horizonte, Minas Gerais durante el año de 2014. Los obstáculos y metas citados fueron analizados por medio de frecuencia relativa y agrupados en categorías. $63,1 \%$ de los usuarios presentaron dificultades referentes a comportamientos psicosociales y culturales, $16,5 \%$ a ambientales y estructurales, 3,3\% económicos, $6,6 \%$ relacionaron a otros factores y $10 \%$ todavía no consideraron tener dificultades relevantes para cuidar de su salud. $60 \%$ de los usuarios demostraron disposición para planeamiento y cumplimiento de metas. Se concluye que el usuario es capaz de buscar soluciones para los obstáculos y tomar decisiones informadas, adecuadas a su salud y contexto de vida, buscando soluciones para el afrontamiento de obstáculos en las prácticas de autocuidado en diabetes.

DESCRIPTORES: Educación en salud; Diabetes Mellitus; Autocuidado; Enfermería. 


\section{INTRODUÇÃO}

A educação para o autocuidado da condição do diabetes fornece ao usuário conhecimentos, atitudes e habilidades necessárias para o desempenho do autocuidado e mudança de comportamento, especialmente dentro das áreas de nutrição e atividade física. O usuário enfrenta barreiras relacionadas aos aspectos comportamental e psicossocial, sendo importante considerar que a multifatorialidade das dificuldades para o desenvolvimento do autocuidado acaba por tornar o processo de cuidar complexo. Buscar compreender as barreiras e, sobretudo, trabalhá-las de acordo com o contexto de vida de cada usuário, é essencial para planejar e sistematizar práticas educativas ${ }^{(1-3)}$.

Neste contexto de incentivar, promover e corresponsabilizar o usuário pela prática do autocuidado, o profissional da área da saúde deve atuar como parceiro no acompanhamento e construção do plano de metas para o autocuidado, motivando o usuário a buscar soluções para as barreiras encontradas na realização das práticas de autocuidado. O usuário é considerado o centro do cuidado e as dificuldades são reconhecidas por ele próprio, o desenvolvimento de soluções se torna palpável, bem como o estabelecimento de metas para superá-las ${ }^{(4-6)}$.

Assim, a academia, em parceria com as unidades básicas de saúde, desenvolve práticas orientadas ao cuidado em diabetes Mellitus por meio da abordagem dialógica. Os dados do presente estudo foram provenientes de visitas domiciliares realizadas por enfermeiros que se basearam no instrumento Protocolo de Mudança de Comportamento em Diabetes Mellitus, o qual prioriza a resolução de problemas, construção de um plano individualizado e definição de metas com avaliação do plano de cuidados ${ }^{(7)}$.

Diante dessa perspectiva o objetivo deste estudo foi identificar barreiras e o cumprimento de metas para o desenvolvimento das práticas de autocuidado em usuários com a condição crônica do diabetes Mellitus tipo 2.

\section{MÉTODO}

Trata-se de um estudo descritivo exploratório, realizado em três unidades básicas de saúde da regional leste do município de Belo HorizonteMG durante o ano de 2014.

A população compreendeu 30 usuários com diabetes tipo 2 determinados por conveniência e que atenderam aos critérios de inclusão do estudo: idade entre 20 e 75 anos, com tempo de diagnóstico da doença menor que vinte anos e não possuir complicações crônicas relacionadas ao diabetes, tais como retinopatia diabética, neuropatias, cardiopatias, nefropatias, entre outras. Para este estudo, considerou-se como variáveis sociodemográficas: sexo, idade, tempo com a doença, ocupação e grau de escolaridade.

A coleta de dados foi realizada por meio de visitas domiciliares, as quais foram realizadas por profissionais enfermeiros guiados pelo instrumento Protocolo Mudança de Comportamento em Diabetes Mellitus. Este questionário é composto por 31 questões abertas, divididas em cinco passos: 1- definição dos problemas; 2- identificação e abordagem dos sentimentos; 3- definição de metas; 4- elaboração do plano de cuidados para conquista de metas e 5- avaliação do plano de cuidados ${ }^{(7)}$.

Cada usuário recebeu uma visita domiciliar, com tempo médio de 40 minutos. As respostas obtidas nas visitas, por sua vez, compuseram um banco de dados e para análise recorreu-se a uma análise de frequência relativa (\%) dos dados tabulados organizados em planilha do Excel 2007 e analisados com apoio do software R versão 3.0.3, com o objetivo de verificar os fatores relacionados às barreiras e ao cumprimento de metas para o desenvolvimento do autocuidado mais citados e assim agrupá-los em categorias, de acordo com as semelhanças. Para descrever o perfil dos usuários segundo as variáveis consideradas também se recorreu a uma tabela de frequências relativas (\%) das variáveis categóricas.

$\mathrm{O}$ estudo atendeu às normas nacionais $\mathrm{e}$ internacionais de ética em pesquisa envolvendo seres humanos, de acordo com a Resolução 466/12(8). O projeto foi submetido ao Comitê de Ética em Pesquisa da Secretaria Municipal de Saúde de Belo Horizonte e obteve parecer positivo sob o número 0024.O.410.203-09a .

\section{RESULTADOS}

As características sociodemográficas dos 30 usuários participantes da pesquisa compreenderam brasileiros, adultos, sendo $74 \%$ idosos $(60$ anos ou mais), em sua maioria do sexo feminino $(70 \%)$, residentes em Belo Horizonte-MG. Cerca de $44,4 \%$ convivia com a condição crônica do diabetes há cinco anos ou menos e no âmbito da renda mensal, obteve-se: $43,3 \%$ (1 a 2 salários/ mês); 26,6\% (2 a 4 salários/mês) e 10\% (5 salários/ 
mês). Observou-se que a maioria dos usuários, $53,3 \%$, não possuía instrução formal ou possuía ensino fundamental incompleto e que somente $6,6 \%$ estudaram até o nível superior completo.

Do total de 30 (100\%) usuários participantes do estudo, $63,1 \%$ apresentaram dificuldades relacionadas aos comportamentos psicossociais e culturais, $16,5 \%$ a ambientais e estruturais, 3,3\% econômicos, $6,6 \%$ relacionaram a outros fatores e, ainda, $10 \%$ não consideraram ter dificuldades relevantes para cuidar da sua saúde, conforme apresentado na Tabela 1.

A alimentação apresentou-se como a principal barreira para o estabelecimento de um plano de cuidados em diabetes, uma vez que $39,9 \%$ (12) dos usuários relataram ter um desejo excessivo de ingerir alimentos ricos em gorduras saturadas e açúcares. Outro fator que merece destaque é o quadro de ansiedade apresentado por $13,3 \%$ dos usuários (4).
No que tange o cumprimento de metas, os usuários detalharam qual ação iriam realizar no âmbito da mudança comportamental, passando por hábitos alimentares, prática de atividades físicas e uso correto dos medicamentos. Em seguida, foi associado o grau de importância atribuído à mudança comportamental, como melhora da autoestima, controle clínico, bem como qualidade de vida.

Percebeu-se que o planejamento para o cumprimento de metas foi significativo, uma vez que $47,05 \%$ dos usuários comprometeram-se com a mudança de hábitos alimentares, 23,53\% com a adoção de atividades físicas regulares e 29,42\% com o uso correto dos medicamentos, o que demonstra que todos os usuários sistematizaram um plano de cuidados de acordo com a sua preferência. Tais dados foram descritos na Tabela 2.

Tabela 1 - Frequência de Fatores Associados às Barreiras Relacionadas à Prática de Autocuidado. Belo Horizonte, MG, Brasil, 2014

\begin{tabular}{|c|c|c|c|c|}
\hline FATORES & $\begin{array}{l}\text { Frequência } \\
\text { Relativa (\%) }\end{array}$ & ESPECIFICAÇÃO & & $\begin{array}{l}\text { Frequência } \\
\text { Relativa (\%) }\end{array}$ \\
\hline \multirow{5}{*}{$\begin{array}{l}\text { COMPORTAMENTOS } \\
\text { PSICOSSOCIAIS E } \\
\text { CULTURAIS }\end{array}$} & \multirow[t]{5}{*}{63,1} & Ansiedade & 4 & 13,3 \\
\hline & & $\begin{array}{l}\text { Esquecimento e dificuldades para tomar/ } \\
\text { administrar a medicação }\end{array}$ & 2 & 6,6 \\
\hline & & $\begin{array}{l}\text { Desejo excessivo de ingerir alimentos ricos } \\
\text { em gorduras saturadas e açúcares }\end{array}$ & 12 & 39,9 \\
\hline & & Sedentarismo & 1 & 3,3 \\
\hline & & Comparecimento a eventos sociais & 2 & 6,6 \\
\hline \multirow[t]{3}{*}{$\begin{array}{l}\text { AMBIENTAIS E } \\
\text { ESTRUTURAIS }\end{array}$} & \multirow[t]{3}{*}{16.5} & $\begin{array}{l}\text { Rotina não propícia para o fracionamento das } \\
\text { refeições }\end{array}$ & 2 & 6,6 \\
\hline & & $\begin{array}{l}\text { Rotina não propícia para a prática de } \\
\text { atividades físicas }\end{array}$ & 1 & 3,3 \\
\hline & & Falta de apoio familiar & 2 & 6,6 \\
\hline ECONÔMICOS & 3,3 & Condição econômica desfavorável & 1 & 3,3 \\
\hline NÃO CITOU & 10 & Declarou não ter dificuldades & 3 & 10 \\
\hline
\end{tabular}

Tabela 2 - Cumprimento de metas para o desenvolvimento do autocuidado. Belo Horizonte, MG, Brasil, 2014

\begin{tabular}{|c|c|c|c|}
\hline $\begin{array}{l}\text { CUMPRIMENTO DE } \\
\text { METAS }\end{array}$ & ESPECIFICAÇÃO & & $\begin{array}{l}\text { Frequência } \\
\text { Relativa (\%) }\end{array}$ \\
\hline \multirow{3}{*}{$\begin{array}{l}\text { DETALHAMENTO } \\
\text { MUDANÇA } \\
\text { COMPORTAMENTAL }\end{array}$} & Mudar hábitos alimentares e tornar-se saudável & 16 & 47,05 \\
\hline & Adotar uma rotina de atividades físicas & 8 & 23,53 \\
\hline & Administrar e ingerir medicação conforme orientação médica & 10 & 29,42 \\
\hline \multirow{3}{*}{$\begin{array}{l}\text { GRAU DE } \\
\text { IMPORTÂNCIA } \\
\text { ASSOCIADO }\end{array}$} & Melhora da autoestima e autoconfiança & 9 & 30 \\
\hline & Melhora do controle glicêmico e resultados de exames & 3 & 10 \\
\hline & Melhora da qualidade de vida e hábitos de vida saudáveis & 9 & 30 \\
\hline OUTROS & Não especificou & 8 & 26,6 \\
\hline
\end{tabular}




\section{DISCUSSÃO}

Dentre os 30 usuários que participaram do estudo, $82,9 \%$ apresentaram dificuldades para a realização do autocuidado, relacionando-as a fatores psicossociais, culturais e ambientais. Em contrapartida, a totalidade da amostra demonstrou disposição para o planejamento e cumprimento de metas, associando-as a um aumento da autoconfiança e melhora da qualidade de vida.

A condição crônica do diabetes está pautada pela educação e o cuidado centrado no usuário com diabetes, em um processo resultante de corresponsabilização juntamente com o profissional da área da saúde e construído por meio de diferentes estratégias educativas. Este processo requer que o profissional da área da saúde e o usuário reconheçam suas tarefas específicas na gestão da condição do diabetes e se comprometam com elas. Para isso, devem-se levar em consideração os aspectos psicossociais, comportamentais e clínicos que envolvem a gestão do cuidado em saúde ${ }^{(9-11)}$.

Autor aponta que os principais obstáculos para a prática do autocuidado incluem o avanço da idade, o nível de escolaridade, renda mensal baixa e falta de apoio familiar. Ainda citam os fatores emocionais como pensamentos angustiantes, dor, desânimo e frustração perante o tratamento ${ }^{(12)}$. Estudo realizado com 24 pessoas com a condição do diabetes, em Ribeirão Preto-SP, demonstrou que a dificuldade para controlar os impulsos alimentares ainda é o principal empecilho a ser superado, seguido da dificuldade em seguir a dieta e em controlar os impulsos negativos, tais como raiva, medo, ansiedade e desânimo ${ }^{(13)}$.

Observou-se no presente estudo que 39,9\% dos usuários apresentaram dificuldades para regrar a alimentação, uma vez que essa se apresenta com destaque em todas as relações sociais, como casamentos, reuniões familiares, religiosas e até políticas. É em volta da mesa que os usuários exercem seu papel de sujeito sociável, reforçando suas relações afetivas. Logo, ter de seguir um plano alimentar saudável, muitas das vezes, pode significar prejuízos emocionais aos usuários e ser uma das principais causas do estresse relacionado a essa condição ${ }^{(14)}$. Tal fato justificaria o resultado de $6,6 \%$ de usuários terem determinado como barreira ao autocuidado o comparecimento a eventos sociais.

Neste cenário, faz-se importante trabalhar a motivação e incentivar o usuário a superar barreiras. Intervenções educativas sistematizadas e que constroem uma parceria entre profissional - usuário, aprofundando-se no encorajamento para a mudança de comportamento e alcance de metas, têm demonstrado resultados favoráveis, trabalhando a motivação extrínseca ${ }^{(1,15)}$.

No entanto, a motivação intrínseca se mostra mais importante na gestão do autocuidado e se torna melhor trabalhada se aliada à construção de planos de metas. Assim como foi feito no presente estudo, é importante garantir que o próprio usuário estabeleça as metas. Para um, pode ser mais significativo ter um bom controle glicêmico por meio de medicamento, enquanto para outro, sentir-se disposto e realizar atividade física é sinônimo de longevidade e qualidade de vida. Ainda, enquanto alguns consideram a liberdade de se cuidarem (ou não), como felicidade ${ }^{(15-16)}$.

É interessante notar que os resultados do presente estudo demonstram que a motivação intrínseca veio seguida do grau de importância atribuído à mudança de comportamento. 30\% dos usuários acreditam que se mudarem o comportamento, melhorarão a autoestima e a confiança em si mesmos. Outros 30\% afirmaram que terão mais qualidade de vida e melhor saúde. As mesmas especificações de barreiras foram apontadas como ações futuras do plano de cuidados e metas, o que sugere que todos querem superar as dificuldades, mas precisam priorizá-las e sistematizá-las.

O Protocolo Mudança de Comportamento em Diabetes Mellitus prioriza a reflexão do usuário e a problematização de seu cotidiano, explorando as barreiras e os sentimentos, sendo possível discutir os problemas e motivar a resolução, visando à mudança comportamental e psicossocial da condição do diabetes. A concordância na elaboração de um plano de metas entre profissional da área da saúde e usuário, é considerada como um pré-requisito para a obtenção de bons resultados no controle glicêmico e satisfação com o tratamento ${ }^{(7)}$.

A vista domiciliar sistematizada e planejada busca construir uma parceria entre profissional e usuário, aprofundando o encorajamento para a mudança de comportamento e alcance de metas para o autocuidado e tem demonstrado resultados efetivos ${ }^{(1,14)}$. No entanto, é importante garantir que o próprio usuário estabeleça as metas para melhor controle glicêmico e qualidade de vida. O estabelecimento de um plano de metas contextualizado com as possibilidades do usuário e um acompanhamento contínuo junto aos profissionais da área da saúde pode vir a auxiliar 
na superação das barreiras relacionadas aos aspectos comportamental e psicossocial ${ }^{(16-17)}$.

Como limitação do estudo cita-se o número amostral, uma vez que diante da complexidade do tratamento da condição crônica do diabetes, diversas barreiras podem surgir, de acordo com os diferentes contextos de vida do usuário.

\section{CONCLUSÃO}

As principais barreiras e metas relacionadas à prática do autocuidado estão relacionadas aos comportamentos psicossociais e culturais. Contudo, dadas barreiras devem ser revistas e trabalhadas com vistas à mudança comportamental e melhor qualidade de vida.

Conclui-se que o usuário é capaz de buscar soluções para as barreiras e tomar decisões informadas, adequadas à sua saúde e contexto de vida, buscando soluções para o enfrentamento de barreiras nas praticas de autocuidado em diabetes.

\section{AGRADECIMENTOS}

À Fundação de Apoio à Pesquisa de Minas Gerais, FAPEMIG, pelo apoio técnico e financeiro.

\section{REFERÊNCIAS}

1. Ahola AJ, Groop PH. Barriers to self-management of diabetes. Diabet Med. 2013; 30(4):413-20.

2. Tang TS, Funnell MM, Brown MB, Kurlander JE. Self-management support in "real-world" settings: an empowerment-based intervention. Patient Educ Couns. 2010; 79(2):178-84.

3. Zoffmann V, Kirkevold M. Realizing empowerment in difficult diabetes care: a guided self-determination intervention. Qual Health Res. 2012; 22(1):103-18.

4. Griffin SJ, Simmons RK, Prevost AT, Williams KM, Hardeman W, Sutton S, et al. Multiple behaviour change intervention and outcomes in recently diagnosed type 2 diabetes: the ADDITION-Plus randomised controlled trial. Diabetologia. 2014; 57(7):1308-19.

5. Rodrigues FFL, Santos MA, Teixeira CRS, Gonela JT, Zanetti ML. Relação entre conhecimento, atitude, escolaridade e tempo de doença em indivíduos com diabetes mellitus. Acta paul. enferm. 2012;25(2):284-90.
7. Funnell MM, Anderson RM. Empowerment and self-management of diabetes. Clinical Diabetes. 2004; 22(3):123-7.

8. Ministério da Saúde (BR). Conselho Nacional de Saúde. Diretrizes e normas regulamentadoras de pesquisa envolvendo seres humanos. Resolução $n$. 466, de 12 de dezembro de 2012. Brasília; 2012.

9. Dixon-Fyle S, Gandhi S, Pallathy T, Spatharou A. Changing patient behavior: the next frontier in healthcare value. Health International. 2012; (12):64-73.

10. Costa JA, Balga RSM, Alfenas RCG, Cotta RMM. Promoção da saúde e diabetes: discutindo a adesão e a motivação de indivíduos diabéticos participantes de programas de saúde. Ciênc. saúde coletiva. 2011; 16(3):2001-9.

11. Torres HC. O empoderamento nas práticas educativas orientadas à autonomia no cuidado em saúde. In: Torres HC, Reis IA, Pagano AS. O empoderamento do pesquisador nas Ciências da Saúde. $1^{\text {a }}$ ed. Belo Horizonte: FALE/UFMG; 2015. p. 1-19.

12. Ong WM, Chua SS, Ng CJ. Barriers and facilitators to self-monitoring of blood glucose in people with type 2 diabetes using insulin: a qualitative study. Patient Prefer Adherence. 2014; 15(8):237-46.

13. Péres DS, Santos MA, Zanetti ML, Ferronato AA. Dificuldades dos pacientes diabéticos para o controle da doença: sentimentos e comportamentos. Rev. Latino-Am. Enfermagem. 2007; 15(6):1105-12.

14. Raaijmakers LGM, Hamers FJM, Martens MK, Bagchus C, De Vries NK, Kremers SPJ. Perceived facilitators and barriers in diabetes care: a qualitative study among health care professionals in the Netherlands.BMC Fam Pract. 2013; 14(114):1-9

15. Silva DMGV, Hegadoren K, Lasiuk G. As perspectivas de donas de casa brasileiras sobre a sua experiência com diabetes mellitus tipo 2. Rev. LatinoAm. Enfermagem. 2012; 20(3):469-77.

16. Abrahão AL. Atenção e cuidado em saúde no ambiente familiar: aspectos da visita domiciliar. Rev APS. 2011; 14(4):472-80.

17. Lange I, Campos S, Urrutia M, Bustamante C, Alcayaga C, Tellez A, et al. Effect of a tele-care model on self-management and metabolic control among patients with type 2 diabetes in primary care centers in Santiago, Chile. Rev Med Chil. 2010; 138(6):729-37.

6. Torres $\mathrm{HC}$, Pace $\mathrm{AE}$, Stradioto MA. Análise sociodemográfica e clínica de indivíduos com diabetes tipo 2 e sua relação com o autocuidado. Cogitare Enferm. 2010; 15(1):48-54. 